\title{
Non-cooperative game theory based energy management systems for energy district in the retail market considering DER uncertainties
}

DOI:

10.1049/iet-gtd.2016.0024

\section{Document Version}

Accepted author manuscript

Link to publication record in Manchester Research Explorer

Citation for published version (APA):

Marzband, M., Javadi, M., Domínguez-García, J. L., \& Moghaddam, M. M. (2016). Non-cooperative game theory based energy management systems for energy district in the retail market considering DER uncertainties. IET Generation, Transmission and Distribution, 10(12), 2999-3009. https://doi.org/10.1049/iet-gtd.2016.0024

Published in:

IET Generation, Transmission and Distribution

\section{Citing this paper}

Please note that where the full-text provided on Manchester Research Explorer is the Author Accepted Manuscript or Proof version this may differ from the final Published version. If citing, it is advised that you check and use the publisher's definitive version.

\section{General rights}

Copyright and moral rights for the publications made accessible in the Research Explorer are retained by the authors and/or other copyright owners and it is a condition of accessing publications that users recognise and abide by the legal requirements associated with these rights.

\section{Takedown policy}

If you believe that this document breaches copyright please refer to the University of Manchester's Takedown Procedures [http://man.ac.uk/04Y6Bo] or contact uml.scholarlycommunications@manchester.ac.uk providing relevant details, so we can investigate your claim.

\section{OPEN ACCESS}




\title{
Non-cooperative game theory based energy management systems for energy district in the retail market considering DER uncertainties
}

\author{
Mousa Marzband ${ }^{\mathrm{a}, \mathrm{b}}$, Masoumeh Javadi ${ }^{\mathrm{c}, \mathrm{d}}$, José Luis Domínguez-García ${ }^{\mathrm{e}}$, Maziar Mirhosseini Moghaddam \\ ${ }^{a}$ The University of Manchester, School of Electrical and Electronic Engineering, Ferranti Building, Manchester, M13 9PL, UK \\ ${ }^{b}$ Dept. of Electrical Engineering, Lahijan branch, Islamic Azad University, Lahijan, Iran \\ ${ }^{c}$ Dep. of Electrical Power Engineering, Guilan Science and Research Branch, Islamic Azad University, Rasht, Iran \\ ${ }^{d}$ Dep. of Electrical Power Engineering, Rasht Branch, Islamic Azad University, Rasht, Iran \\ ${ }^{e}$ Catalonia Institute for Energy Research (IREC), Jardins de les Dones de Negre 1, 08930 Sant Adrià de Besòs, Barcelona, Spain
}

\begin{abstract}
In this paper, a general framework for implementing a retail energy market based on the Nikaido-Isoda/relaxation algorithm is proposed as an electricity market structure with large distributed energy resources (DERs) penetration and demand side management (DSM) of consumers. Moreover, the consumers are able to participate in the market as prosumers (i.e., producer and consumer at the same time). By considering the related uncertainties, the DERs can maximize their expected payoff or profit by undertaking strategies through the price bidding strategy, based on the proposed structure, considering Nash equilibrium. The results show the effectiveness and accuracy of the proposed framework in determining the optimal power set-points of players participating in the market to achieve the objectives.
\end{abstract}

Keywords: Optimal energy management, electricity market, energy district, demand side management, Nikaido-Isoda function and relaxation algorithm, uncertainty

\section{Introduction} deregulated market where providers and consumers may be active participants in the electricity price pool. The evolu- ${ }_{-30}$ tion of power systems into more controllable systems leads ${ }_{31}$ to a significant increase in the number of market players. 32 These players are presently able to freely enter the market ${ }_{33}$ and access new economic opportunities provided by the mar- 34 ket. The participation of the market actors guides the mar- 35 ket toward cost reduction and increasing market reliability, 36 increasing the profit of its players $[1,2]$. All market par-37 ticipants present an offer which is used for computing the ${ }_{38}$ real market clearing price (MCP). Market deregulation laws 39 have been adjusted given possible technical problems such 40 as system disconnections as well as economic decisions (e.g., 41 unacceptable offers). The strong effect of technical grid constraints may lead to economic decisions impacting the be- ${ }_{43}$ haviour of the consumers. The electricity market is defined by complex concepts such as unhealthy competition, strat- ${ }_{45}$ egy interference, unbalanced information and the possibil- ${ }_{46}$ ity of multi-phase equilibrium [3-5]. Traditional economic ${ }_{47}$ modelling methods have faced problems in confronting these ${ }_{48}$ concepts. The smart competitive models based on agents, ${ }_{49}$ are an attractive candidate for modelling and simulating such problems. Each agent states an independent participant with ${ }^{50}$

Email address: mousa .marzband@manchester.ac.uk, Tel. $+44(0) 7442555372$ Corresponding author (Mousa Marzband)
6

7

cor kets, the number of generators is significantly higher than the number of sellers, and most of the research done in the past years has been focused on the supply side. In [6-10], different scenarios of playing individually or cooperatively were simulated and the results showed that it is of great benefit to cooperate, but the free-rider problem may arise. In [11] an improved dynamic programming algorithm is developed that requires fewer operations and less memory than the original one. Given the large number of agents, this property is a major drawback because agents usually have limited time and would not be able to wait until the end of the execution of the algorithm.

In the global competitive market, electricity buyers are no longer price takers since they are able to influence the market by using different bidding strategies, as well as cooperating with other buyers. Therefore, it is necessary to develop and investigate individual and cooperative strategies of electricity buyers. However, most of the research efforts concentrate on the generation of the test cases and how to solve the constraint problem; whereas research on demand side has not been sufficiently forthcoming.

In this market structure, the agents of both sides of supply and consumption continuously adapt their strategies according to their objective function.

An evolutional model based on the agent can simulate a 54 
for the generators and a countless number of consumers in 12 a competitive market have been studied in [12] by using 13 Monte-Carlo sampling for estimating the behaviour of rivals ${ }_{14}$ [13] has focused on minimizing the locational marginal price15 of purchasers by using different evolutional algorithms andr16 adding decision modules based on game theory. The mech ${ }_{117}$ anisms of the solution of this method are related to fairness $x_{118}$ efficiency and stability in distributing the payoffs among the 19 agents. Furthermore, widespread efforts have been devoted 20 to the area of coalition formation. The method of interesti21 investigated in the researches has focused on dividing the $\mathbf{2}_{22}$ agents inside the coalition such that the sum of payoffs in all 23 of the coalition become maximum, which optimal coalition 124 structure. The alliance strategy of buyers has been studied in 25 [13] and it has been shown that the buyers could lower theili26 costs by evolving their group sizes and memberships.

Economic operations of future residential distribution sys 128 tems with high participation of distributed electricity pro129 sumers with various non-convex objective function includ ${ }_{130}$ ing generation, storage and responsive load offers is pre ${ }_{131}$ sented in previous literature [14-21]. However, previouslyi32 published works have been mainly concentrated on the con $_{133}$ trol and operation problem of individual energy district (ED) 134 $_{4}$ with particular attention to frequency stability and reliability/35 improvement, and thus, coupled operation in systems based.36 on producers and prosumers is still considered as an emerg ${ }_{137}$ ing area of research. Furthermore, while the existing stud ${ }_{138}$ ies have focused on simplifying market models by introduc ${ }_{139}$ ing only a small number of participants, the proposed modehao can easily be implemented over a grid with a large number of purchasers and sellers. This is done by completely taking into account the technical and economic constraints and, further, can be easily developed for consideration of different 42 market models. The residential customers here are not onlyias electricity consumers but can through local exploitation of 44 distributed generators, energy storage devices and their dis $\mathbf{1 4 5}$ tributable loads become electricity supplies. The proposedia6 framework provides significant economic incentives for in ${ }_{147}$ stalling and commissioning their distributed renewable gen ${ }_{148}$ erators (e.g, wind turbine (WT) in small scale and photo 149 voltaic (PV) panel installed over the roof of houses). $\quad{ }_{150}$

It is expected that in the future, EDs became a commonis1 entity within the distribution grid, which will have the ca152 pability of import and export energy depending on the sit153 uation. Thus, it may have the chance on participate in the 54 electricity market if several of them apply together (cooper ${ }_{155}$ ate). The paper aims to present a methodology for ensuring 56 profits for micro-prosumers (producers and consumers at the ${ }_{57}$ same time as autonomous houses can be [15]). The conceptiss relies on the concept of aggregator [15], where different au ${ }_{159}$ tonomous houses, forming an energy district (ED), may be60 big enough to participate within the energy market. Since ${ }_{161}$ different ED are interacting together (cooperating) they are62 sharing load needs for minimizing the power imported frombs the main grid. Moreover, such concept allows the EDs sellingi64 the power excedence in the market due to their bigger size 165 The earnings from such market participation must be shared;06 this paper presents a methodology based on game-theory for EDs participation on the retail market and a earning sharing procedure for maximizing the earnings of each ED participating.

Furthermore, it is desirable to develop non-cooperative behaviour of players for maximizing the benefits. The cooperation and pricing strategies of power sellers in a deregulated market have been studied using algorithms based on noncooperative game theory in a framework based on agents. Complex systems have not allowed analysis of larger number of participants; but in this paper the proposed model can easily be implemented over a grid with a large number of buyers taking into account of technical and economic constraints and can be easily extended to other market models. The innovations of this paper can be listed as follows:

1. Identification and formulation of the functionalities of generation units and distributed electricity prosumers in the future residential distribution systems considering uncertainties;

2. Development of package-based software, using a novel non-cooperative game-theoretic methodology and uncertainty algorithms to further analyse the behaviours of the key market participants in different scenarios, and to clear the retail electricity market price;

3. Investigation of the impact of distributed electricity prosumers on the economic operations of the future residential distribution systems;

4. Conduct of numerical case study to evaluate and validate the proposed framework.

\section{The proposed stochastic market clearing procedure}

The proposed structure for electricity markets presents a solution for enabling high share participation of distributed generation. This market aims to reduce electricity price, maximizing generation and consumption players profit through cooperation. The considered framework provides this possibility for the exploiters of distribution system and household customers to select in a proper method for their energy supply resource by noting different choices, such as selecting a wide range of renewable energy resources based on final electricity cost. In the proposed framework, the household consumers play a relevant role in the exploitation and management of distributed energy resources (DER) (distributed generation (DG) resources, energy storage (ES)) and also responsive load demand (RLD). The DERs can also cooperate for obtaining more profit. On the other hand, the consumers while participating with the producers for managing their demand attempt to reduce final electricity price. The proposed structure is shown in Figure 1. The proposed structure has four stages as follows:

Stage 1: The amount of energy produced by WT, PV and non-responsive load (NRL) demand are forecasted.

Stage 2: The scenarios of energy consumed by nonresponsive load (NRL) demand, and the energy generated by both WT and PV are generated using the information obtained in the previous stage. Moreover, generation uncer- 
tainty is carried out through stochastic programming for con $_{202}$ sidering generation variability.

Stage 3: The expected values of generation and consump-204 tion powers are randomly selected for participation in theos game theory based on different scenarios and their occur 200 rence probability. Then, the Nash equilibrium point in theor market electricity price is obtained considering local andos global constraints.

Stage 4: The optimum capacity for market participationp10 is obtained by using relaxation algorithm and Nikaido-Isodær11 function for a set of players in line with reaching maximump12 profit. During this stage by noting the optimum Nash equilib 213 rium obtained previously, the pay-off function of each player can be computed.

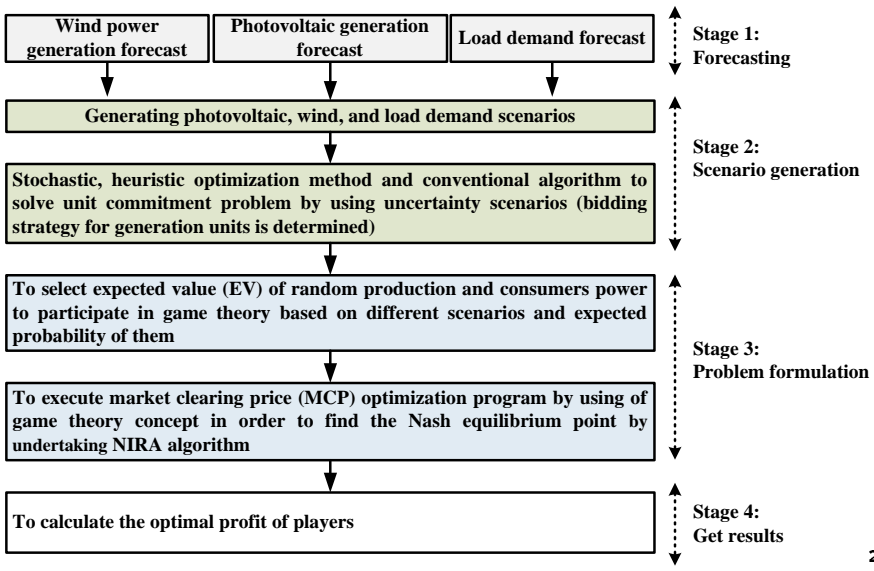

Figure 1: The proposed market structure implementation process

\section{ED system under study}

For testing the proposed method, an isolated ED is developed in MATLAB software. The specifications of the system ${ }^{220}$ and the control plans for the DERs are detailed in [18-21] 221 Figure 2 illustrates the system under study.

222

The ED under study includes a collection of productionz23 resources (WT, PV and MT), ES and consumers as nonz24 responsive load (NRL) and RLD. The predicted data for load demand, WT and PV taken from references [22]. The collec 225 tion of production resources is defined as player $\# 1$ and $\mathrm{RLD}_{226}$ as player \#2. The cost coefficients (a,b and c), retail electric ${ }_{227}$ ity price $(\theta$ and $\beta)$, the NRL load $(\zeta)$ and supply price $\left(\pi^{\mathrm{ES}-}{ }_{228}\right.$ and $\pi^{\mathrm{ES}+}$ ) are summarized in Table 1.

The cost coefficients (a, b, c), profit and the price of power supply of players \#1 and \#2 have been summarized in Table 1.

For evaluating the performance of the retail energy marke (REM) based on the Nikaido-Isoda/relaxation (REM-NIRA) algorithm, a sensitivity analysis is performed by applying the following scenarios:
1. Scenario \#1: sudden increase (10\%) the power generated by renewable resources;

2. Scenario \#2: the initial value of state-of-charge (SOC) set at maximum value;

3. Scenario \#3 (normal condition): the initial value of SOC at average value;

4. Scenario \#4: the initial value of SOC set at minimum value;

5. Scenario \#5: sudden decrease (10\%) the power generated by renewable resources.

The value of SOC in the initial conditions is adjusted equal to its average value (i.e. 50\%) in scenarios \#1, \#3 and \#5.

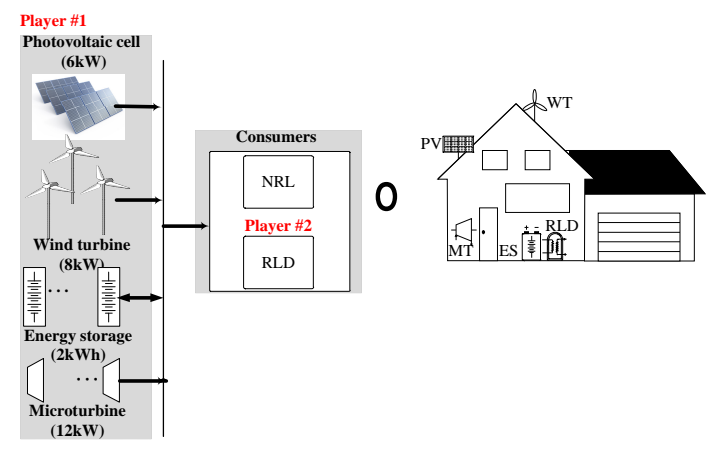

Figure 2: schematic of the system under study

\section{Problem formulation}

In this section, the mathematical formulation of the problem is presented by considering the key components of participation in the retail market. The general framework is easily expandable and adjustable with other electricity distribution systems with high levels of customers participation.

\subsection{Objective functions and problem constraints}

The proposed market key components include two players: the generation collective (Player \#1) and consumption collective (Player \#2) in ED. Each one presents its own objective function, as presented in the following.

\subsubsection{Objective functions}

Player \#1: The generation resources in the grid under study is made up of MT, WT, PV and ES during discharging mode. The profit obtained from production collections (Player \#1) can be calculated at $t^{\text {th }}$ hour by

$$
\begin{aligned}
& \max \mathrm{J}_{\mathrm{t}}^{\text {Player }-1}=\mathbb{R}_{\mathrm{t}}^{\text {Player-1 }}-\mathbb{C}_{\mathrm{t}}^{\text {Player }-1} \\
& \mathrm{t} \in\{1,2, \cdots, 24\}
\end{aligned}
$$

\section{$\mathbb{R}$} where $\mathbb{R}_{t}^{\text {Player-1 }}$ and $\mathbb{C}_{t}^{\text {Player-1 }}$ are respectively the revenue and cost of Player \#1 at time t. The revenue (i.e. $\mathbb{R}_{t}^{\text {Player-1 }}$ ) can be defined as:

$$
\mathbb{R}_{t}^{\text {Player-1 }}=\lambda\left(P_{n}\right) \times\left[P_{t}^{M T}+P_{t}^{W T}+P_{t}^{P V}+P_{t}^{E S-}-P_{t}^{n}\right]
$$


Table 1: Cost coefficients and the profits of players \#1 and \#2

\begin{tabular}{cccccccc}
\hline $\mathrm{a}$ & $\mathrm{b}$ & $\mathrm{c}$ & $\theta$ & $\beta$ & $\zeta$ & $\pi^{\mathrm{ES}}-$ & $\pi^{\mathrm{ES}+}$ \\
\hline$\$ / \mathrm{kW}^{2} \mathrm{~h}$ & $\$ / \mathrm{kWh}$ & $\$ / \mathrm{h}$ & $\$ / \mathrm{kWh}$ & $\$ / \mathrm{h}$ & & $\$$ & $\$$ \\
$6 \mathrm{e}^{-6}$ & 0.01 & 0 & 0.001 & 3.4 & 60 & 0.125 & 0.145 \\
\hline
\end{tabular}

where $P_{t}^{M T}$ is the MT output power (kW), $P_{t}^{W T}$ WT output power $(\mathrm{kW}), \mathrm{P}_{\mathrm{t}}^{\mathrm{PV}}$ is the PV system output power $(\mathrm{kW})$ and $P_{t}^{E S-}$ is the ES discharge power at $t^{\text {th }}$ hour $(\mathrm{kW})$.

For sake of simplicity, the retail electricity price is considered the same for all the players following a singular distri265 bution system [23].

$$
\lambda\left(P_{n}\right)=\left(-\theta \times P_{t}^{n}\right)+\beta, \theta \geqslant 0
$$

where $\theta$ and $\beta$ are load demand curve coefficient, $\lambda$ the iden ${ }_{267}$ tical electricity price in $\$ / k W h$ and $P_{t}^{n}$ total ED system givenes load demand in $\mathrm{kW}$ at the $\mathrm{t}^{\text {th }}$ hour. The cost function (i.e 269 $\mathbb{C}_{t}^{\text {Player-1 }}$ ) is expressed in $\$$ by.

$$
\mathbb{C}_{\mathrm{t}}^{\text {Player-1 }}=\mathbb{C}_{\mathrm{t}}^{\mathrm{MT}}+\mathbb{C}_{\mathrm{t}}^{W T}+\mathbb{C}_{\mathrm{t}}^{\mathrm{PV}}+\mathbb{C}_{\mathrm{t}}^{\mathrm{ES}-}+\mathbb{C}_{\mathrm{t}}^{\mathrm{ES}+}
$$

The generators WT and PV are as non-distributable units and their output power is dependent upon climatic conditions. The cost of renewable resources generation is negligible compared to other resources; as a result, $\mathbb{C}_{t}^{W T}=0$ and $^{272}$ $\mathbb{C}_{t}^{P V}=0$ have been considered. The MT generation cost has $s^{273}$ been formulated as a quadratic function as follows.

$$
\mathbb{C}_{\mathrm{t}}^{M T}=\mathrm{a} \cdot\left(\mathrm{P}_{\mathrm{t}}^{M T}\right)^{2}+\mathrm{b} \cdot \mathrm{P}_{\mathrm{t}}^{M T}+\mathrm{c}, \quad \mathrm{a} \geqslant 0
$$

where $a, b$ and $c$ are the coefficients of cost function for MT. The ES charge and discharge costs are also computed through the following relations $[24,25]$.

$$
\begin{aligned}
& \mathbb{C}_{\mathrm{t}}^{\mathrm{ES}-}=\pi^{\mathrm{ES}-} \times \mathrm{P}_{\mathrm{t}}^{\mathrm{ES}-} \\
& \mathbb{C}_{\mathrm{t}}^{\mathrm{ES}+}=\pi^{\mathrm{ES}+} \times \mathrm{P}_{\mathrm{t}}^{\mathrm{ES}+}
\end{aligned}
$$

Each one of the above mentioned players make their deci $_{283}$ sions depending on local and global constraints.

\subsubsection{Local and global constraints}

Equilibrium constraint: the equilibrium of the power gen 287 erated by Player \#1 must be equivalent to the total power consumed by load demand satisfying:

$$
P_{t}^{M T}+P_{t}^{W T}+P_{t}^{P V}+P_{t}^{E S-}=P_{t}^{n}+P_{t}^{E S+}+P_{t}^{R L D}
$$

Player \# 1: MT is exploited during the given time t under the following conditions.

$$
\underline{\mathrm{P}}^{M T} \leqslant \mathrm{P}_{\mathrm{t}}^{M T} \leqslant \overline{\mathrm{P}}^{M T}
$$

where $\underline{P}^{M T}$ and $\bar{P}^{M T}$ are respectively the minimum and maximum output power when MT is active (kW). The ED system, exploits its WT and PV by noting the expected mathematical value resulting from uncertainty scenarios dependent on the following constraints.

$$
\begin{aligned}
& 0 \leqslant P_{t}^{W T} \leqslant E V_{t}^{W T} \\
& 0 \leqslant P_{t}^{P V} \leqslant E V_{t}^{P V}
\end{aligned}
$$

where $E V_{t}^{W T}$ and $E V_{t}^{P V}$ are respectively the WT and PV mathematical expected value at the $t^{\text {th }}$ hour in $\mathrm{kW}$ which is calculated from the product of the probability of the occurrence of each one of the uncertainty scenarios by the value of that scenario according to the following relations.

$$
\begin{aligned}
E V_{\mathrm{t}}^{W T} & =\sum_{s=1}^{\mathrm{N}_{\mathrm{s}}} \rho_{\mathrm{t}}^{W \mathrm{~T}, \mathrm{~s}} \times \mathrm{P}_{\mathrm{t}}^{W T, \mathrm{~s}} \\
E V_{\mathrm{t}}^{\mathrm{PV}} & =\sum_{\mathrm{s}=1}^{\mathrm{N}_{\mathrm{s}}} \rho_{\mathrm{t}}^{\mathrm{PV}, \mathrm{s}} \times \mathrm{P}_{\mathrm{t}}^{\mathrm{PV}, \mathrm{s}}
\end{aligned}
$$

where $N_{s}$ is the number of uncertainty scenarios and $\rho_{t}^{W T, s}$ and $\rho_{t}^{\mathrm{PV}, \mathrm{s}}$ are the possibility of occurrence of each one of the scenarios WT and PV at the $t^{\text {th }}$ hour and $P_{t}^{W T, s}$ and $P_{t}^{P V, s}$ are the WT and PV power values in the related scenario. The proposed algorithm uses ES in t by noting the charge or discharge mode by following the constraints bellow [23].

$$
\begin{aligned}
& 0 \leqslant \mathrm{P}_{\mathrm{t}}^{\mathrm{ES}-} \leqslant \overline{\mathrm{P}}^{\mathrm{ES}-} \\
& 0 \leqslant \mathrm{P}_{\mathrm{t}}^{\mathrm{ES}+} \leqslant \overline{\mathrm{P}}^{\mathrm{ES}+}
\end{aligned}
$$

where $\overline{\mathrm{P}}^{\mathrm{ES}-}$ and $\overline{\mathrm{P}}^{\mathrm{ES}+}$ are respectively the ES maximum power in $\mathrm{kW}$ at ES discharge or charge mode. For preventing excessive charge and discharge when ES SOC reaches its highest and lowest mode, ES charges its state to stand-by mode.

$$
\underline{\mathrm{SOC}} \leqslant \mathrm{SOC}_{\mathrm{t}} \leqslant \overline{\mathrm{SOC}}
$$




$$
\mathrm{SOC}_{\mathrm{t}+1}-\mathrm{SOC}_{\mathrm{t}}=\left(\mathrm{P}_{\mathrm{t}}^{\mathrm{ES}+}-\mathrm{P}_{\mathrm{t}}^{\mathrm{ES}-}\right) \times \Delta \mathrm{t} / \mathrm{ES}_{\mathrm{Tot}}
$$

where $\mathrm{SOC}_{\mathrm{t}}$ is the state of charge of ES. $\overline{\mathrm{SOC}}$ and $\mathrm{SOC}$ are respectively the minimum and maximum state of ES in the charge and discharge mode. $E S_{\text {Tot }}$ is ES capacity in kWh and $\Delta t$ is the time interval.

Player \#2 is the grid made up of RLD and NRL loads. The relation between RLD and NRL during each time interval satisfies the following relation.

$$
0 \leqslant P_{t}^{R L D} \leqslant \zeta \times P_{t}^{n}
$$

$\zeta$ is a coefficient of NRL load.

\subsection{Mathematical modelling of $P V, W T$ and load demand un-} certainty

Since the market is based on predicted data and generation units are variable, uncertainty must be considered. For conforming the predicted data with reality, probabilistic models are used.

\subsubsection{Consumed load demand uncertainty modelling}

Load uncertainty can be modelled using normal distribu ${ }_{328}^{322}$ tion curve and by using average parameters and standard $\mathrm{de}_{{ }_{329}}$ viation. The average value in the load normal curve distribu- ${ }_{330}$ tion is equal to the predicted load during each time inter ${ }_{\overline{3}_{31}}$ val. The standard deviation is obtained from the load predic ${ }_{332}$ tion method based on experience and previous consumption ${ }_{333}$ pattern. To simplify mathematical calculations, the normal ${ }_{334}$ distribution can be divided into several sections showing the $\mathrm{e}_{35}$ load occurrence probability with the value equal to the mean value of that section. In this study the normal probability $y_{36}$ distribution curve according to Figure 3 is used [26, 27].

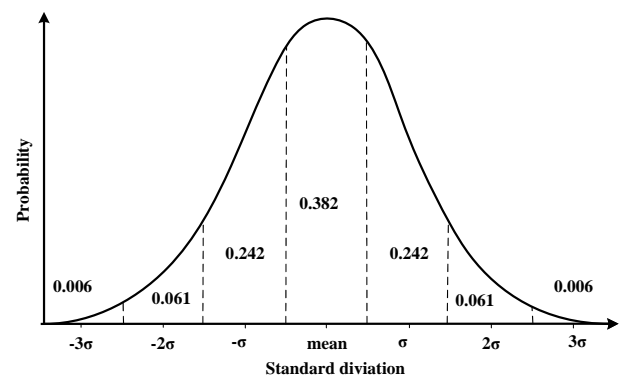

Figure 3: Seven pieces normal probability distribution curve

\subsubsection{WT uncertainty modelling}

Noting that the wind speed is a random variable, calcula tion of wind speed variability during 24 hours Weibull distriзs1 bution is used. The average value of this distribution, is the wind speed prediction datum. The Weibull distribution curves2 can also be divided into several separate sections. The pos 353 sibility of occurrence of each interval is determined through $\mathrm{B}_{\mathbf{5} 4}$ the corresponding wind speed with the mode of each section ${ }_{355}$ The wind speed probability distribution curve in this study356

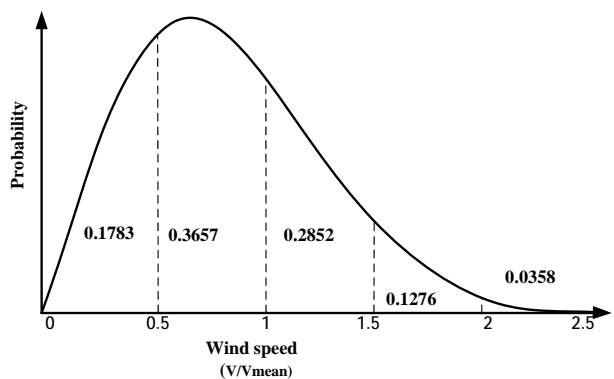

Figure 4: Wind speed probability distribution

is divided in the five pieces distribution density function as shown in Figure $4[28,29]$.

Wind output power is determined from power function based on wind speed according to the following relation.

$$
P_{t}^{W T}(v)= \begin{cases}\left(\frac{P_{r}}{V_{r}-V_{c i}}\right)\left(v-V_{c i}\right) & \text { if } V_{c i} \leqslant v \geqslant V \\ P_{r} & \text { if } V_{r} \leqslant v \geqslant V_{c o} \\ 0 & \text { others }\end{cases}
$$

where $\mathrm{P}_{t}^{W \mathrm{~T}}(v)$ is the WT output power, $v$ is the wind speed, $P_{r}$ WT nominal power, $V_{r}$ wind nominal speed and $V_{c i}$ turbine cut-in speed and $V_{c o}$ is the turbine cut-off speed. If the turbine generation starts at the speed $V_{c i}$; the output power will increase proportional to speed increase from $V_{c i}$ to $V_{r}$ and the nominal power $P_{r}$ is generated when the wind speed is variable between $V_{r}$ and $V_{c o}$. Because of security reasons, the turbine will turn off at speed $V_{c o}$ and the output power will be zero at a speed outside the mentioned limits.

\subsubsection{PV uncertainty modelling}

The amount of solar radiation which reaches earth, in addition to the external daily and annual motion of the sun, depends on the geographic position (length, width and height) and climatic conditions (for example cloud cover). The PV surfaces output power is dependent on the amount of solar radiation of the PV panel surface. The solar radiation hourly distribution can be divided into five sections similar to Weibull distribution model for wind speed which is illustrated in Figure 5 [30]. PV system power distribution is obtained based on radiation distribution. The PV system output power is calculated as follows:

$$
P_{t}^{P V}=A_{C} \cdot \eta \cdot I_{t}^{\beta}
$$

where $A_{C}$ is the area of array surface $\left[\mathrm{m}^{2}\right], \mathrm{I}_{\mathrm{t}}^{\beta}$ is the amount of solar radiation over a surface with $\beta$ slop relation to the horizon surface $\left[\mathrm{kWm}^{-} 2\right], \eta$ is the efficiency of PV system at the realistic reporting conditions.

\subsection{Nikaido-Isoda function}

In the proposed electricity market, all the players compete with each other regulating their output power and load demand. The nature of the electricity market fits into game theory with n-person in a non-cooperative game in which each 


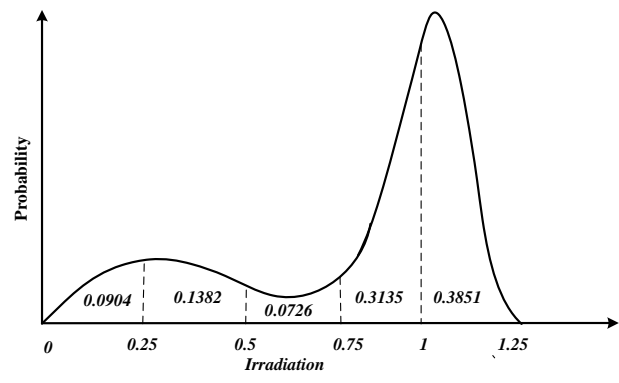

Figure 5: Solar radiation probabilistic distribution player is after maximizing its own profit through a distributed decision-making process. This non-cooperative game with $n_{300}$ players can be solved repeatedly by using the game theory special method (Nikaido-Isoda function and Relaxation algorithm). In a non-cooperative game with $n$ players, each player may have conflict of interests with others [23]. All of them try to maximize their welfare by regulating their strate- ${ }^{402}$ gies. In a mathematical form, a game with $\mathrm{n}$ players is de ${ }^{403}$ fined with tertiary structure $i \in N, N, X_{i}, \Theta_{i}$. Each $i^{\text {th }}$ playere4 belongs to a set of $N=1,2, \cdots, n$ players. $X_{i}$ is the strat ${ }^{405}$ egy space of the $i^{\text {th }}$ player. The set of collective strategies isoo defined as $X=X_{1} \times X_{2} \times \cdots \times X_{n}$. Each player noting itsor special strategy, selects an action through a vector $x_{i}$. The ${ }^{00}$ set of collective action is defined as $x=\left(x_{1}, x_{2}, \cdots, x_{n}\right)$. $\Theta_{1}^{409}$ is the $\mathrm{i}^{\text {th }}$ player pay-off function who calculates the obtain ${ }^{410}$ able profit of that player through regulating its strategy by $y^{11}$ noting the others strategy space. The expression $y_{i} \mid x$ shows ${ }^{12}$ the element $\left(x_{1}, \cdots, x_{i-1}, y_{i}, x_{i+1}, \cdots, x_{n}\right)$ and states that the $\left(x_{1}, \cdots, x_{i-1}, x_{i+1}, \cdots, x_{n}\right)$ player has adopted the behaviour while the other players $i^{\text {th }}$ are present in the game. The Nash equilibrium for each $\mathrm{i}^{\text {th }}$ player is defined as $x^{\star}=$ $\left(x_{1}^{\star}, \cdots, x_{n}^{\star}\right)$.

$$
\Theta_{i}\left(x^{\star}\right)=\max _{\left(x_{i} \mid x\right) \in X} \Theta_{i}\left(x_{i} \mid x\right)
$$

In other words, a Nash equilibrium exists if always for all the players and all the $x_{i}$ holds $i \in N, \Theta_{i}\left(x_{i}^{\star} \mid x^{\star}\right) \geqslant$ $\Theta_{i}\left(x_{i}^{\star} \mid x^{\star}\right)$. Noting that the strategies of other players is constant, no player can improve its payoff function by the unilateral changes of its strategy. The relations 1 and 8 can respectively be considered as pay-off function in the form of relation 23. The Nikaido-Isoda function is defined as follows:

$$
\psi(x, y)=\sum_{i=1}^{n}\left[\Theta_{i}\left(y_{i} \mid x\right)-\Theta_{i}(x)\right]
$$

The expression $\left[\Theta_{i}\left(y_{i} \mid x\right)-\Theta_{i}(x)\right]$ at the right side of the ${ }^{419}$ above relation shows improvement in the $i^{\text {th }}$ pay-off function ${ }^{420}$ when the player changes its behaviour from $x_{i}$ to $y_{i}$, while the strategies of other players stays without change. $x^{\star}$ is $a_{423}^{422}$ Nash normalized equilibrium if:

$$
\max _{\left(x^{\star}, y\right) \in X} \psi\left(x^{\star}, y\right)=0
$$

When a Nikaido-Isoda function reaches zero, no player can increase its pay-offs by the unilateral changes of its strategy. One Nash normalized equilibrium is also a Nash equilibrium point if:

$$
X=X_{1} \times X_{2} \times X_{n}
$$

Therefore, the Nikaido-Isoda function can approximately approach the Nash equilibrium point. Because of this, optimum response function is stated as Nikaido-Isoda function maximum result.

$$
Z(x)=\arg \max _{y \in X} \psi(x, y) x, Z(x) \in X
$$

argmax is for maximum argument and states a set of $\mathrm{x}$ variables for which $\psi(x, y)$ reaches its maximum value. Each player tries unilaterally to maximize its profit.

\subsection{Relaxation algorithm}

Relaxation algorithm improves the optimum response function, respectively until when it becomes convergent to a Nash equilibrium point $x^{\star}$. The initial guess $x^{0}$ is selected based on the information obtained from uncertainty scenarios and the defined planning of units participation. In this paper, $x^{0}$ is the vector of the expected mathematical values obtained from uncertainty scenarios $E V_{t}^{M T}, E V_{t}^{W T}, E V_{t}^{P V}$, $E V_{t}^{E S}$ and $E V_{t}^{R L D}$ which the values $E V_{t}^{W T}$ and $E V_{t}^{P V}$ are calculated through relations 13 and 14 and noting the relations below the other components of vector $x^{0}$ can be determined.

$$
\mathrm{EV}_{\mathrm{t}}^{\mathrm{MT}}=\sum_{s=1}^{\mathrm{N}_{\mathrm{s}}} \rho_{\mathrm{t}}^{\mathrm{MT}, \mathrm{s}} \times \mathrm{P}_{\mathrm{t}}^{\mathrm{MT}, \mathrm{s}}
$$

where $E V_{t}^{M T}, \rho_{t}^{M T, s}$ and $P_{t}^{M T, s}$ are respectively the values of mathematical expected value, possibility of the occurrence of scenario and MT power in the related scenario.

$$
\begin{aligned}
\mathrm{EV}_{\mathrm{t}}^{\mathrm{ES}+} & =\sum_{s=1}^{\mathrm{N}_{\mathrm{s}}} \rho_{\mathrm{t}}^{\mathrm{ES}+, \mathrm{s}} \times \mathrm{P}_{\mathrm{t}}^{\mathrm{ES}+, \mathrm{s}} \\
\mathrm{EV}_{\mathrm{t}}^{\mathrm{ES}-} & =\sum_{s=1}^{\mathrm{N}_{\mathrm{s}}} \rho_{\mathrm{t}}^{\mathrm{ES}-, \mathrm{s}} \times \mathrm{P}_{\mathrm{t}}^{\mathrm{ES}-, \mathrm{s}} \\
\mathrm{EV} V_{\mathrm{t}}^{\mathrm{ES}} & =\mathrm{EV}_{\mathrm{t}}^{\mathrm{ES}+}-\mathrm{EV}_{\mathrm{t}}^{\mathrm{ES}-}
\end{aligned}
$$

where, $E V_{t}^{E S+}, \rho_{t}^{E S+, s}$ and $P_{t}^{E S+, s}$ are the values of mathematical expected value, probability of occurrence of scenario and scenario power in the ES charging mode and in this order $E V_{t}^{E S-}, \rho_{t}^{E S-, s}$ and $P_{t}^{E S-, s}$ are the values of expected mathematical value, possibility of occurrence of scenario and scenario power in the ES discharging mode. $E V_{t}^{E S}$ is the value of mathematical expected value of the sum of charging and discharging modes of ES.

$$
E V_{t}^{R L D}=\sum_{s=1}^{N_{s}} \rho_{t}^{R L D, s} \times P_{t}^{R L D, s}
$$


where $E V_{t}^{R L D}, \rho_{t}^{R L D, s}$ and $P_{t}^{R L D, s}$ are respectively the values of RLD mathematical expected value, probability of scenario occurrence and RLD consumed power in the related scenario. The next step, is the respected improvement of optimum response function by using the relaxation algorithm.

$$
\left\{\begin{array}{c}
x^{k+1}=\left(1-\alpha_{k}\right) x^{k}+\alpha_{k} Z\left(x^{k}\right) \\
k=0,1,2, \cdots
\end{array}\right.
$$

where $\mathrm{k}$, is the iteration step and $\alpha_{\mathrm{k}}$ is the weight allocated to $x^{k}$ and $Z\left(x^{k}\right)$ is for obtaining new $x^{k+1}$ in the k next step. The optimum response function, as long as shutdown condition is satisfied as follows, is updated repeatedly.

$$
\max _{\left(x^{k}, y\right) \in X} \psi\left(x^{k}, y\right) \leqslant \epsilon
$$

$\epsilon$ is a small value defined by the user for controlling the convergence rate. The convergence of relaxation algorithm is guaranteed with a constant $\alpha_{k}$ for a convex-concave function, when $\alpha_{k}$ satisfies the following conditions.

$$
\sum_{k=0}^{\infty} \alpha_{k}=\infty, \quad 0 \leqslant \alpha_{k} \leqslant 1, \alpha_{k} \rightarrow 0, k \rightarrow 0
$$

During each iteration $k$, an optimum value $\alpha_{k}$ is found through solving the following optimum response function:

$$
\alpha_{k}^{\star}=\arg \min _{0 \leqslant \alpha_{k} \leqslant 1}\left[\max _{y^{k} \in X} \psi\left(x^{k+1}\left(\alpha_{k}\right), y^{k}\right)\right]
$$

After several iterations, the tranquillity algorithm converges to an $x^{\star}$ Nash equilibrium point. That is $\mathrm{x}$ is a compact convex set of Euclidean space. Optimum response function $Z\left(x^{k}\right)$ is a singular and continues value over $x^{s}$. In addition to this, the Nikaido-Isoda function (i.e. $\psi(x, y)$ ) is a weak convex-concave and quadratic continues differentiable function. In the other hand, a unique equilibrium point exist such that the relaxation algorithm can become convergent in it [31].

\section{REM-NIRA algorithm}

The flowchart for implementing the REM-NIRA algorithm is presented in Figure 6. As observed in this figure, the algorithm is made up of three main units including uncertainty, MCEMS unit and NIRA unit. The proposed REM-NIRA algorithm is illustrated by a Pseudo-code in Algorithm 1. The method for implementing them is explained in the following sections.

\subsection{TOAT unit}

This unit is proposed for including uncertainty related to load demand and the effect of the change of environmental conditions on the power generated by renewable resources by using the Taguchi's orthogonal array testing

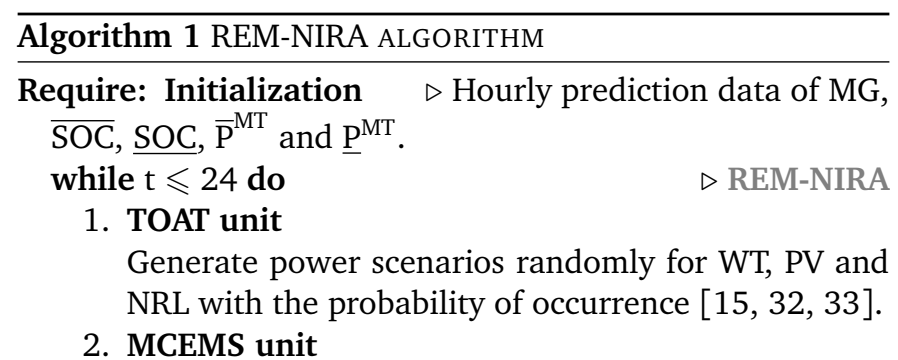

Determine $P_{t}^{\mathrm{MT}, \mathrm{s}}, \mathrm{P}_{t}^{\mathrm{ES}-\mathrm{s}}, \mathrm{P}_{\mathrm{t}}^{\mathrm{ES}+, \mathrm{s}}, \mathrm{P}_{\mathrm{t}}^{\mathrm{RLD}, \mathrm{s}}, X_{t}^{\mathrm{ES}, \mathrm{s}}[20,22]$;

3. Calculate $\mathrm{EV}_{\mathrm{t}}^{\mathrm{WT}}$ (Eq. 13), $\mathrm{EV}_{t}^{\mathrm{PV}}$ (Eq. 14), $\mathrm{EV}_{t}^{\mathrm{MT}}$ (Eq. 27), $\mathrm{EV}_{t}^{\mathrm{ES}}$ (Eq. 30), $\mathrm{EV}_{\mathrm{t}}^{\mathrm{RLD}}$ (Eq. 31), in addition to determining $\mathrm{X}_{\mathrm{t}}^{\mathrm{ES}}$.

4. Define $x^{0}$ using the results obtained in step 3;

5. NIRA unit

(a) Determine the parameters required by the REMNIRA algorithm (the dimension vector of players, upper/lower bound players, maximum number of iterations, selecting the $\alpha$ optimization step, tolerance)

(b) Define $\Phi_{i}$ (Eq. 1-8);

(c) Define the players constraints (Eq. 9-19);

(d) Form $\Psi(x, y)$ (Eq. 23);

(e) Calculate $x^{\star}$ (Eq. 24);

(f) Maximize $\Psi(x, y)$ and form $Z(x)$ (Eq. 26)

(g) Apply the relaxation algorithm and improving $\mathrm{Z}(\mathrm{x})$ by Eqs. 32 and 35 until the stooping condition (Eq. 24);

(h) Determine output data;

(i) Determine $x^{\star}$;

(j) Calculate the values of the players profit based on $x^{\star}$ (Eq. 1-8);

(k) Determine $\mathrm{SOC}_{\mathrm{t}+1}$;

end while

return Determine the optimum capacity and profit of the all players. 


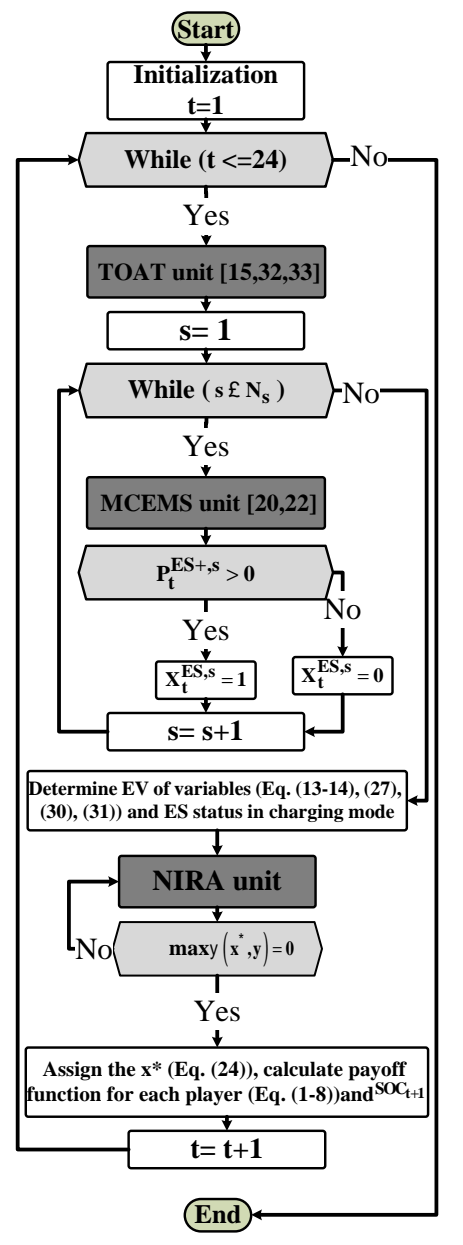

Figure 6: The proposed algorithm for implementing REM-NIRA

(TOAT) method. The TOAT method in [15] is produced a robust solutions for consideration of uncertainties within multiple electrically coupled EDs. The TOAT method ensures that the testing scenarios provide good statistical information with a minimum scenario number in the uncertain operating ${ }_{988}$ space, which significantly reduces the testing burden $[32]_{480}$ For additive and quadratic models, TOAT has been proven to have the ability to select optimally representative scenarios for testing from all possible combinations [33]. Compared with Monte Carlo simulation, TOAT provides much smaller ${ }_{493}$ testing scenarios and leads to shorter computing time. $\mathrm{De}_{494}^{-{ }_{494}}$ spite of its ability, it has only been adopted as an optimization algorithm to solve the economic dispatch problem in the ${ }_{496}^{495}$ power system field [34, 35].

The existing uncertainties in the discussed problem have been implemented with the scenarios formed according to 98 Figure 7. The proposed structure include three main stages $s_{499}$ as follows:

Stage 1: Selecting the orthogonal matrix noting the numbero1 of uncertainties existing in the system;

Stage 2: Create $n$ values for load demand using a normakos distribution, for WT using a Weibull distribution and for504 PV using a Weibull distribution and the radiation equa ${ }_{505}$ tion;

Stage 3: Calculate the possibility of the occurrence of created scenario by using the distribution functions related to load, WT and the radiation function related to PV.

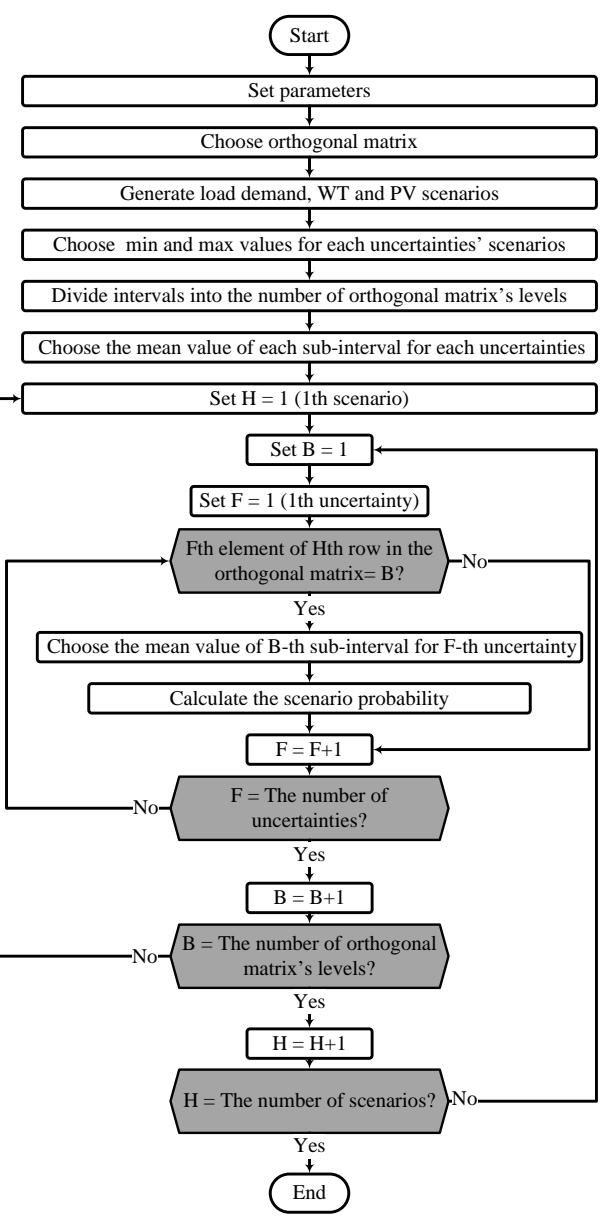

Figure 7: TOAT unit

\subsection{MCEMS unit}

For determining the set-point of the power related to generation resources and consumption modified conventional energy management system (MCEMS) algorithm presented in [20] is used. In fact this unit determines the initial values for all the variables defined based on technical and economic constraints related to each element for the NIRA unit. Details about the method of implementation and the quality of the performance of these units has been explained completely in [20] and is outside the scope of this paper.

\subsection{NIRA unit}

This unit has two important duties with two sub-problems including 1- Maximizing Nikiado-Isoda function 2- Applying the relaxation algorithm and improving the optimum response function. Both of these duties are repeatedly followed by the NIRA unit when the difference of the optimum response function in relation 26 between two adjacent iterations becomes small enough. After initial value giving, first 
the relation 23 must be maximized for finding $y_{i}$ by notingsi the local and global constraints 9 to 19. Then, gradually, the6r solution obtained from relation 23 in the first sub-problem ${ }_{563}$ converges to a new stable state which shows the desired re sult in solving the first sub-problem. After the intermediate 65 solution found in the first sub-problem, we go to the $\sec _{560}$ ond sub-problem so the relaxation algorithm is executed. $\mathrm{In}_{\mathbf{6} 67}$ the second sub-problem, the relaxation algorithm is executed6s in the relation 32 such that it improves the solution spacese $x^{k}$. Then for updating $x^{k}$, the relaxation algorithm is exe $e_{570}$ cuted through relations 32 and 35 . If the values Nikaidos71 Isoda reaches zero, no player can unilaterally improve it\$72 pay-off function. On the other hand, a balanced (approxisz mate) response for electricity market settlement by following 74 the global and local constraints (relations 9-19) is found. Byb75 the repeated improvement of the optimum response function 576 in relation 32, gradually the values of the pay-off function ${ }_{77}$ of all the players converges to an approximate equilibrium ${ }_{78}$ point. Noting the found balanced response through contin ${ }_{579}$ uous iterating rings, the electricity market price for an EDso system with several customers can be settled.

\section{Result and Discussion}

Figures 8(a)-8(c) show data resulting from applying uncertainty (TOAT unit) for load demand inputs, WT and PV during 24 hour time interval. As observed, significant changes over the value of generated power by renewable resources by applying uncertainty under different scenarios is observable. Especially in scenario \#1 the value of the increase of power generated by WT in some of time intervals is more than $18 \%$ of its value in other scenarios. As observed in the Figure 9(a), despite the application of different scenarios the value of load demand power remains constant during the whole system operation. The real life experimental data carried out from $[18,19]$ are used to WT, PV and NRL profiles. The main effect of system conditions change shows itself over the value of the power consumed by DR. As it is observed from Figure 9(a), during the early hours of the day very small changes in the value of DR power is observed and during the day until 15:00 clock. Significant change in its value has not been observed. After this time, if the SOC initial value is set at minimum value (scenario \#3), the system will observe sudden decrease with the value of $20 \%$ from its maximum value in this time interval. The reason for that is the REM-NIRA algorithm tries to reduce $30 \%$ of maximum power generated by MT resulting in much less excess power is generated by generation resources compared with other scenarios, as shown in Figure 9 (b). During the remaining hours of the day, algorithm in all of the applied scenarios puts MT in service with more capacity of generation. In scenario \#1 with the increase of the value of the power generated by renewable resources, the value of SOC about $54 \%$ of time intervals has increased relative to normal conditions. Despite the increase of its value during the system daily performance, and it reaching its maximum value among applied scenarios (about 70\%), its value at the end of the day has reached its minimum value. Its reason is that the value of generated excess power is spent supplying DR power, and during $92 \%$ of time intervals its value is more than scenario $\# 3$. In scenario $\# 2$, only $4 \%$ of time intervals the value of SOC is more than its value during normal conditions; however $42 \%$ of time intervals the value of DR power has reduced relative to normal conditions. This fact shows that after complete discharge during the system performance initial time, the ES SOC value during most time intervals remains constant at minimum value and the excess power generated is used mainly for supplying DR until the end of system operation, during which the proposed algorithm has decided to change ES in all of the scenarios except scenario \#1, as depicted in Fig. 10(a). Regarding the total consumed power (TCP) generated by the grid under study as it is observed from Figure 11, in more than $96 \%$ of the time intervals its value has become more than its value in scenario \#3 which the difference of the consumed power in fact is spent supplying DR and ES in more intervals. This condition is scenario \#2 has reached about $54 \%$ of scenario \#3 and its reason can be pointed out as more ES discharging times (10\%) (according to Figure 10(a)) under this scenario relative to scenario \#3 despite the $42 \%$ increase in supplying DR.

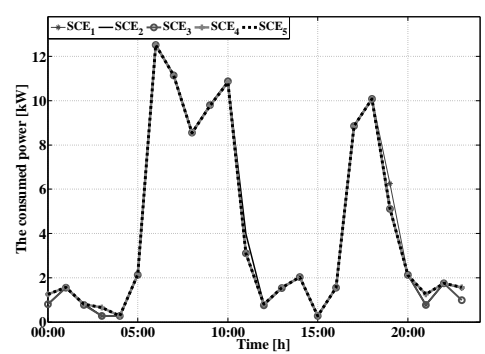

(a) Load demand profile

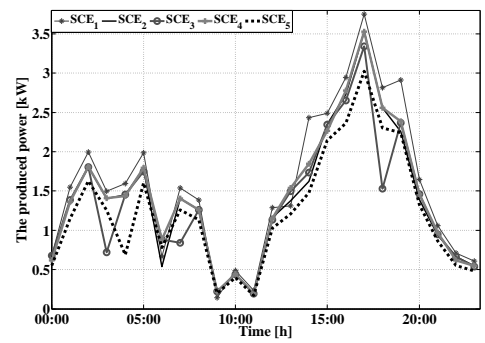

(b) WT profile

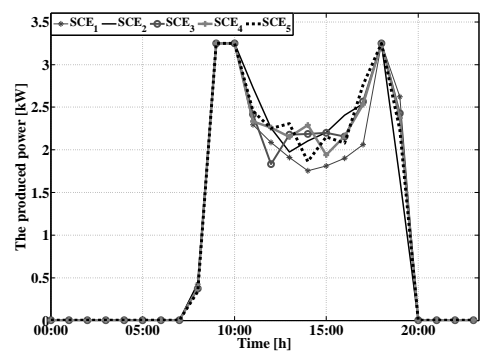

(c) PV profile

Figure 8: The results obtained by REM-NIRA algorithm 


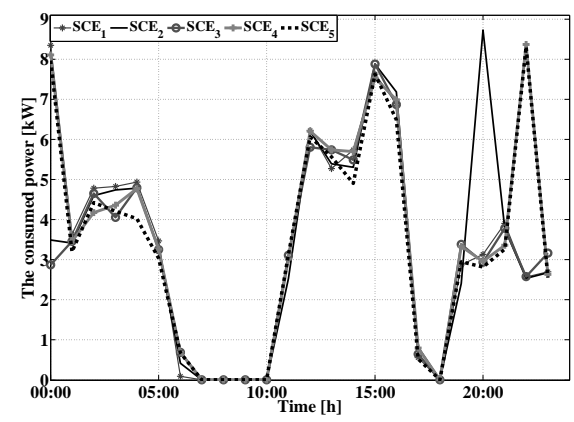

(a) DR profile

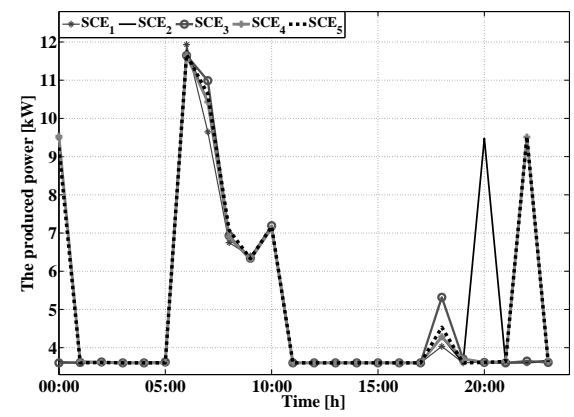

(b) MT profile

Figure 9: The results obtained by REM-NIRA algorithm

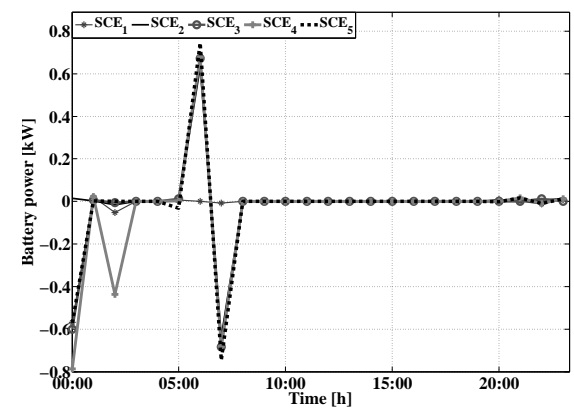

(a) ES profile

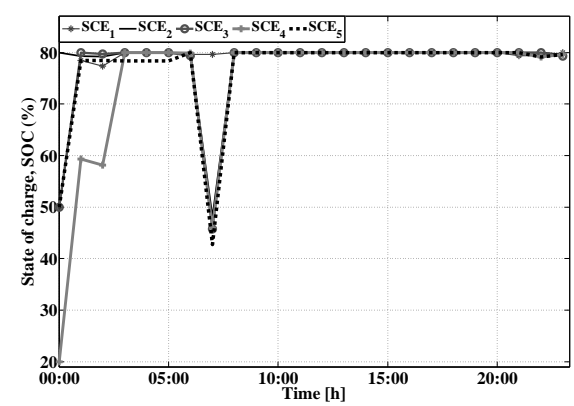

(b) SOC profile

Figure 10: The results obtained by REM-NIRA algorithm

For reaching convergence in the algorithm, each sequence15 of the measures of step (i.e. $\alpha_{k}$ ) may be selected between 16 each iteration for converging to the Nash equilibrium point ${ }_{617}$ The measure of adequate steps may improve by trial and er ${ }_{618}$ ror while its value changes between the interval $0 \leqslant \alpha \leqslant 1.619$

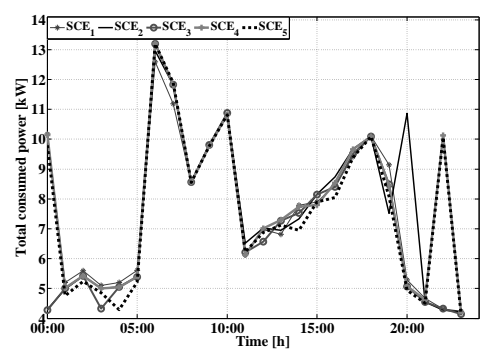

Figure 11: TCP profile

In many cases, it has been observed that by using constant step $\alpha_{\mathrm{s}}=0.5$ it is quickly guided to the convergence value. Although the size of the steps can also be selected by the user, however in the proposed REM-NIRA algorithm the possibility of finding $\alpha_{k}$ optimum value during the simulation operations with the aid of a mechanism exists. For this purpose by defining the optimum value control parameter, $\alpha_{k}$ is also determined by the algorithm for the next step. The optimum value of $\alpha_{k}$ during the time intervals has been shown in Figure 12.

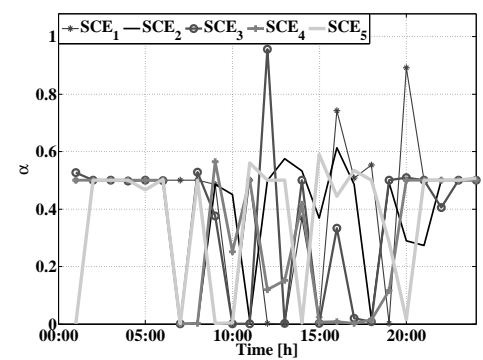

Figure 12: The optimum value of $\alpha_{k}$

Figures 13(a) and 13(b) show the values of converged pay-off function for consuming and generating players in the best iteration during each time interval. As it is observed from the figures, the values of pay-off functions of the players in all time intervals have become the same. The values of pay-off functions of all of the players gradually converge to the Nash equilibrium point by periodically updating the optimum response function (relation 26). In the Nash equilibrium point, noting that the strategies of other players are constant, no player can improve its pay-off function by the unilateral changes of its strategy.

\section{Conclusions}

In this paper, a method has been proposed for implementing a market based on a competitive framework by noting obtaining maximum profit by DER units and minimum MCP by the consumers. The method presented has applied a relaxation algorithm, Nikaido-Isoda function and non-cooperative game theory. Both generation and the adequate distribution of renewable energy resources for consumers of electricity distribution system are main advantages of REM-NIRA algorithm. The customers in the proposed structure play an 


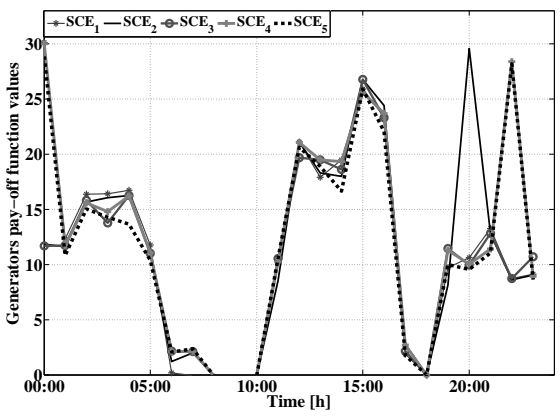

(a) Pay-off function of generation resources

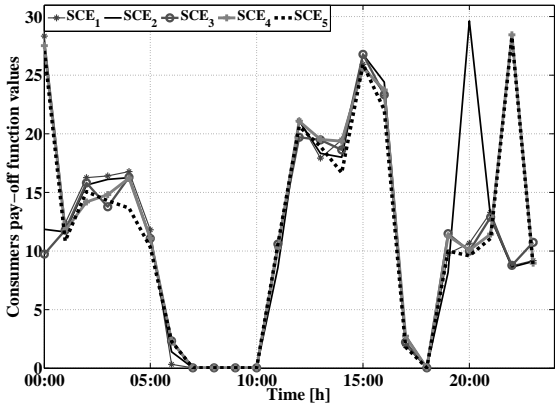

(b) Pay-off function of consumers

Figure 13: The profile of the pay-off function of consumers and generation resources

important role through exploiting and managing DERs, ES\$877 and RLD loads locally, in contrast with conventional marketirs structures. This algorithm provides the possibility of combin 679 ing grid and other renewable energy resources constraints asso well as presenting the ability to implement generation andssi demand prediction curves. The proposed ED including DER ${ }_{982}$ ES and responsive loads resources and by considering the ef $f_{83}$ fect of load demand uncertainties and the variations of DER\$s4 output power. It is observed that in the presented structure ${ }_{985}$ effective motivations for DER units to supply load and corss operate with each other for obtaining more profit and, ins87 the case of consumers, for cooperating with each other for reducing electricity cost. The developed model can also be used as a useful tool for analysing market power and also ${ }_{88}$ for evaluating the strategic behaviour of effective agents in a competitive electricity market. Moreover, it can be used by ${ }_{68}$ the system independent operator for increasing market effi ${ }_{6890}$ ciency. For continuing the work, the proposed structure folro1 systems with multi-ownership is under development in whichfo2 each owner has several DERs, ES and separate responsive loads. In the proposed structure, the existing DER resource $\$ 95$ in each grid enter game independently with the consumerø96 and follow their goal for obtaining more profit while satisfy ${ }_{698}^{697}$ ing all the constraints defined. Furthermore, a market struc ${ }_{699}^{698}$ ture based on non-cooperative game theory is also developedoo among different owners for increasing their obtained profito relative to independent optimisation. Therefore, the contri ${ }^{702}$ butions and aims of the presented paper can be summarized ${ }_{704}^{703}$ as follows:
1. Presenting a survey relevant to retail competition model in electricity market;

2. Proposing a market model for trading distributed renewable energy by guaranteeing fairness among noncooperative players;

3. Using a static non-cooperate game theory to model the interaction among different DERs, ESs, and loads;

4. Studding the competition of DER and RLD in a deregulated market;

5. Applying the NIRA methodology to find an electricity market equilibrium in order to clearing the retail electricity market price;

6. Analyzing the players's behavior by using the concept of Nash equilibrium as a solution in the multi-agent interaction problems;

7. Implementing a retail energy market with full prosumers' participation through probabilistic methodology;

8. Implementing demand-side energy management in order to use as a method for further balancing the supply and demand of power systems with high levels of renewable energy generation and controlling a residential energy storage system;

9. Trying to increase the contribution of green energy in electricity supply, by high-performance green buildings;

10. Promoting distributed energy resources such as energy efficiency, DER, energy storage devices, and renewable energy resources, increasing the number of DER installations through encouraging the costumers;

11. Encouraging demand side participation in the electricity market;

12. Decreasing problems caused by distributed generation and increase the value of intermittent energy in the market by combining distributed generation with energy storage and demand response;

13. Employing the supply function bidding mechanism to model electricity market;

14. Applying the developed RLD scheme as an energy resource of electricity retailers;

\section{References}

[1] M. Shahidehpour, H. Yamin, Z. Li, Market Operations in Electric Power Systems: Forecasting, Scheduling, and Risk Management, Vol. 9, John Wiley \& Sons, 2002.

[2] M. A. Kopaee, M. Manbachi, H. Khezeli, M. Ghazizadeh, A. RahimiKian, Netpms: an internet-based power market simulator for educational purposes, IET Generation, Transmission \& Distribution, 6 (2012) 472-81.

[3] H. Nafisi, V. Farahani, H. A. Abyaneh, M. Abedi, Optimal daily scheduling of reconfiguration based on minimisation of the cost of energy losses and switching operations in microgrids, IET Generation, Transmission \& Distribution, 9 (2015) 513-22.

[4] J. Ruiz, J. Wang, C. Liu, G. Sun, An outer-approximation method for security constrained unit commitment, IET Generation, Transmission \& Distribution, 7 (2013) 1210-18.

[5] L. Tesfatsion, Agent-based computational economics: A constructive approach to economic theory, Agent-Based Computational Economics, (2005) 55 
[6] M. Carvalho, J. P. Pedroso, J. Saraiva, Electricity day-ahead markets 77 Computation of nash equilibria, Journal of Industrial and Managemen $\$ 78$ Optimization, 11 (3) (2015) 985-98.

[7] K. Lee, Strategy equilibrium in stackelberg model with transmissiorrso congestion in electricity market, Journal of Electrical Engineering ands Technology, 9 (1) (2014) 90-97.

[8] J. Molina, J. Zolezzi, J. Contreras, H. Rudnick, M. Reveco, Nash 783 cournot equilibria in hydrothermal electricity markets, IEEE Transacz84 tions on Power Systems, 26 (3) (2011) 1089-101.

[9] J. Krawczyk, Numerical solutions to coupled-constraint (or gener 780 alised nash) equilibrium problems, Computational Management Sciz87 ence, 4 (2) (2007) 183-204.

[10] S. Uryasév, R. Rubinstein, On relaxation algorithms in computation of 89 noncooperative equilibria, IEEE Transactions on Automatic Controly9o 39 (6) (1994) 1263-67.

[11] M. Tesser, A. Paǵes, N. Nabona, An oligopoly model for medium-termr92 power planning in a liberalized electricity market, IEEE Transactions $\mathbf{9 3}$ on Power Systems, 24 (1) (2009) 67-77.

[12] F. Wen, A. David, Optimal bidding strategies for competitive generaz95 tors and large consumers, International Journal of Electrical Power \& $\$ 96$ Energy Systems, 23 (1) (2001) 37-43.

[13] J. Contreras, M. Klusch, J. Krawczyk, Numerical solutions to nash尹98 cournot equilibria in coupled constraint electricity markets, IEEFrog Transactions on Power Systems, 19 (1) (2004) 195-206.

14] M. Sanjari, G. Gharehpetian, Game-theoretic approach to cooperativeso1 control of distributed energy resources in islanded microgrid considso2 ering voltage and frequency stability, Neural Computing and Applicaвоз tions 25 (2) (2014) 343-51.

[15] M. Marzband, N. Parhizi, M. Savaghebi, J. M. Guerrero, Distributedøo 5 smart decision-making for a multi-microgrid system based on a hier 806 archical interactive architecture, IEEE Transactions on Energy Conversion, PP (99) (2015) 1-12.

[16] M. Marzband, N. Parhizi, J. Adabi, Optimal energy management for stand-alone microgrids based on multi-period imperialist competition algorithm considering uncertainties: experimental validation, International Transactions on Electrical Energy Systems, (2015) 1-15.

[17] M. Marzband, E. Yousefnejad, A. Sumper, J. L. Domínguez-García, Real time experimental implementation of optimum energy management system in standalone microgrid by using multi-layer ant colony optimization, International Journal of Electrical Power \& Energy Systems, 75 (2016) 265-74.

[18] M. Marzband, F. Azarinejadian, M. Savaghebi, J. M. Guerrero, An optimal energy management system for islanded microgrids based on multiperiod artificial bee colony combined with markov chain, IEEE systems journal, (99) (2015) 1-11.

[19] M. Marzband, M. Ghadimi, A. Sumper, J. L. Domínguez-García, Experimental validation of a real-time energy management system using multi-period gravitational search algorithm for microgrids in islanded mode, Applied Energy, 128 (2014) 164-74.

[20] M. Marzband, A. Sumper, A. Ruiz-Álvarez, J. L. Domínguez-García, B. Tomoiagă, Experimental evaluation of a real time energy management system for stand-alone microgrids in day-ahead markets, Applied Energy, 106 (2013) 365-76.

[21] M. Marzband, A. Sumper, J. L. Domínguez-García, R. Gumara-Ferret, Experimental validation of a real time energy management system for microgrids in islanded mode using a local day-ahead electricity market and MINLP, Energy Conversion and Management, 76 (2013) 314-22.

[22] M. Marzband, Experimental validation of optimal real-time energy management system for microgrids, Phd thesis, Departament d'Enginyeria Elèctrica, EU d'Enginyeria Tècnica Industrial de Barcelona, Universitat Politècnica de Catalunya (2013).

[23] W. Su, A. Q. Huang, A game theoretic framework for a next-generation retail electricity market with high penetration of distributed residential electricity suppliers, Applied Energy, 119 (2014) 341-50.

[24] M. Marzband, A. Sumper, Implementation of an optimal energy management within islanded microgrid, International Conference on Renewable Energies and Power Quality (ICREPQ), Cordoba, Spain, 2014.

[25] M. Marzband, A. Sumper, M. Chindriş, B. Tomoiagă, Energy management system of hybrid microgrid with energy storage, The International Word Energy System Conference (WESC), Suceava, Romania, 2012.
[26] R. Billinton, a. Bonaert, a. Koivo, Power System Reliability Evaluation, 5th Edition, Vol. 1, Gordon and Breach, New York, 1971.

[27] M. Fotuhi-Firuzabad, M. Rastegar, A. Safdarian, F. Aminifar, Probabilistic home load controlling considering plug-in hybrid electric vehicle uncertainties, in: Reliability Modeling and Analysis of Smart Power Systems, Springer India, 2014, pp. 117-32.

[28] A. Daniel, A. Chen, Stochastic simulation and forecasting of hourly average wind speed sequences in jamaica, Solar Energy, 46 (1) (1991) $1-11$.

[29] B. Borowy, Z. Salameh, Optimum photovoltaic array size for a hybrid wind/PV system, IEEE Transactions on Energy Conversion, 9 (3) (1994) 482-88.

[30] G. Tina, S. Gagliano, S. Raiti, Hybrid solar/wind power system probabilistic modelling for long-term performance assessment, Solar Energy, 80 (5) (2006) 578-88.

[31] B. S. Y. Jin, Trade-off between performance and robustness: An evolutionary multiobjective approach, Springer Berlin Heidelberg, (2003) 237-51.

[32] R. Chatthaworn, S. Chaitusaney, Transmission network expansion planning considering renewable energy target with taguchi's orthogonal array testing, IEEJ Transactions on Electrical and Electronic Engineering, 9 (2014) 588-99.

[33] H. Yu, C. Chung, K. Wong, Robust transmission network expansion planning method with taguchi's orthogonal array testing, IEEE Transactions on Power Systems, 26 (3) (2011) 1573-80.

[34] M. Bounou, S. Lefebvre, X. Do, Improving the quality of an optimal power flow solution by taguchi method, International Journal of Electrical Power \& Energy Systems, 17 (2) (1995) 113-18.

[35] M. Klusch, A. Gerber, Dynamic coalition formation among rational agents, Intelligent Systems, 17 (3) (2002) 42-7. 